\title{
The Temperature-Compensated Goodwin Model Simulates Many Circadian Clock Properties
}

\author{
Peter Ruoff† And Ludger Rensing \\ $\uparrow$ School of Technology and Science, Stavanger College, P.O. Box 2557 Ullandhaug, \\ $N$-4004 Stavanger, Norway and \$Department of Biology, University of Bremen, \\ P.O. Box 3304 40, D-28334 Bremen, Germany
}

(Received on 16 August 1995, Accepted on 15 September 1995)

\begin{abstract}
Goodwin's model oscillator, which consists of a negative feedback loop within a single gene expression pathway $(1963,1965)$, was modified in order to demonstrate temperature compensation, a characteristic property of circadian oscillators. Temperature effects were introduced into the oscillatory mechanism by means of the Arrhenius equation. This term may provide a simplified estimate of the complicated temperature-dependence of actual cellular processes. Temperature compensation within the oscillatory mechanism was achieved by balancing the effects of different temperatures on amplifying processes and on stabilizing processes (antagonistic balance; Ruoff, 1994). Apart from temperature compensation, the modified Goodwin oscillator showed other characteristic properties of circadian clocks including:

(i) temperature entrainment even by temperature cycles of rather low amplitude $\left(1^{\circ} \mathrm{C}\right)$,

(ii) phase shifts by temperature pulses or steps of different signs (up or down) and different amplitudes, giving rise to phase response curves similar to those reported, for example, in Neurospora crassa (Francis \& Sargent, 1979; Rensing et al., 1987; Nakashima, 1987).

A single change in one of the reaction rates of the model oscillator not only caused a change in the period length but also abolished temperature compensation. This closely corresponds to the effects observed after a single point mutation of the clock gene $\left(\mathrm{frq}^{7}\right)$ in Neurospora crassa (Aronson et al., 1994b). Limit cycle oscillations were damped at permanently low temperatures, however the period of the damped oscillations was still temperature compensated.
\end{abstract}

(C) 1996 Academic Press Limited

\section{Introduction}

One of the characteristic properties of circadian clocks is the temperature-compensation of their frequency (Pittendrigh \& Caldarola, 1973; Balzer \& Hardeland, 1988; Edmunds, 1988; Lakin-Thomas et al., 1990). This observation implies the presence of a control mechanism that keeps the circadian period unchanged at different but constant temperatures. This mechanism is assumed to compensate for temperature-induced changes in the processes involved in the clock mechanism. Temperature-

$\dagger$ Author to whom correspondence should be addressed. E-mail: Ruoff@hsr.no. compensation is necessary for a correct timing of the "hands" of the clock, i.e., the numerous clock-controlled processes and the correct determinations of light or dark periods in photoperiodic responses.

How is temperature-compensation possible in view of the fact that most enzyme-catalysed processes are known to be strongly dependent upon temperature? A variety of theories and specific models have been proposed to solve this problem (Hastings \& Sweeney, 1957; Pavlidis et al., 1968; Njus et al., 1974; Rössler et al., 1975). We have previously argued that even chemical oscillators themselves contain all the necessary components for generating temperaturecompensation. If the effect of temperature on the 
amplifying and on the inhibiting (stabilizing) parts of the oscillatory reactions are balanced, the oscillator's period length will be temperature compensated. This principle, termed antagonistic balance (Ruoff, 1992, 1994), has been applied to oscillators which are usually not temperature-compensated, e.g. the Brusselator (Prigogine \& Lefever, 1968), the Kauffman-Wille model (Kauffman \& Wille, 1975), and the Oregonator (Field \& Noyes, 1974). In this paper we extended this principle to a model of the circadian clock.

A recent detection of a feedback loop between clock gene activity and the amount of its product as a basic mechanism of the circadian clock (Aronson et al., 1994a; Zeng et al., 1994) has made it attractive to use Goodwin's model oscillator (1965), which is based on a similar feedback mechanism. Aronson et al. (1994b) concluded that both changes originate from the same point mutation (amino acid exchange) from the observation that some period length mutants (for example $\mathrm{frq}^{7}$ in Neurospora crassa) also show defects in temperature compensation. Therefore, we used the Goodwin oscillator and the concept of antagonistic balance to test whether a single change of a rate constant, for example a slower degradation of the clock protein, may cause changes in both period length and temperature-compensation. This is actually observed in our model.

We further tested, whether the temperaturecompensated Goodwin oscillator can be entrained by periodic temperature changes and whether the oscillator can be phase shifted similar to circadian clocks when perturbed by single temperature steps or pulses. These characteristic properties of circadian clocks were also simulated by the model.

As in chemical oscillators (Ruoff, 1992) we used the Arrhenius equation to describe the influence of temperature on reaction rates in spite of the fact that reaction rates in biological systems are controlled by a number of factors, such as the activity of enzymes, the concentration of the substrates (and products), their transport rates, etc., which are often actively adapting to different temperatures. It is reasonable to assume, however, that the activation energy in the Arrhenius equation can be used as a gross estimate of the temperature dependence of reaction rates in biological systems.

\section{The Model}

The model studied is defined by the following three differential equations (Goodwin 1963, 1965; Drescher et al., 1982; Rensing \& Schill, 1985, 1987):

$$
\begin{gathered}
\frac{\mathrm{d} X}{\mathrm{~d} t}=\frac{k_{1}}{Z^{9}+1}-k_{4} X \\
\frac{\mathrm{d} Y}{\mathrm{~d} t}=k_{2} X-k_{5} Y \\
\frac{\mathrm{d} Z}{\mathrm{~d} t}=k_{3} Y-k_{6} Z
\end{gathered}
$$

The $k_{\mathrm{i}}$ 's are rate constants of processes $R i$ [Fig. 1(a)] and $X, Y$, and $Z$ represent chemical components and their respective concentration values. In molecular terms, $X$ may represent mRNA that codes for a
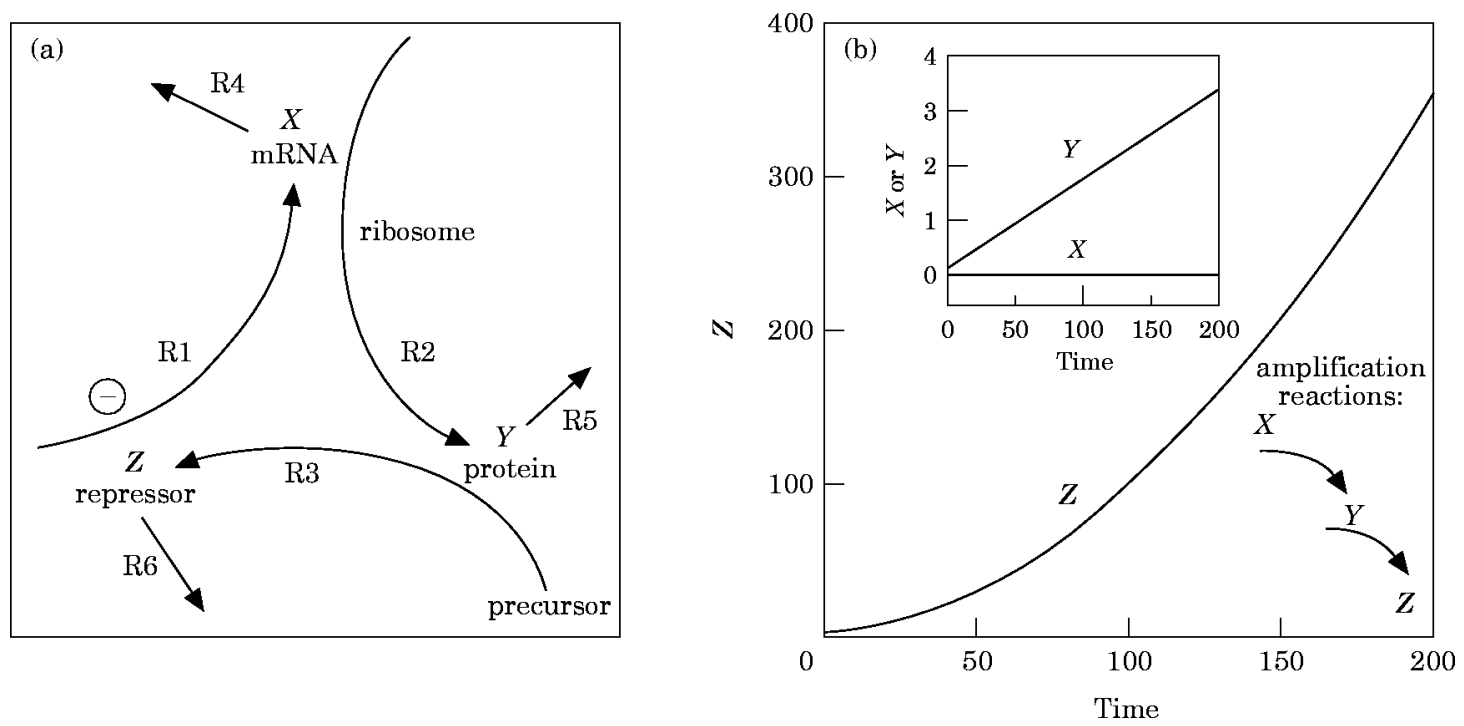

FIG. 1. (a) Inhibition reaction R1, amplifying reactions R2, R3, and stabilizing reactions R4, R5, and R6 in the Gooodwin model. (b) The amplifying reactions lead to a destabilization of the system, which is obtained by setting the rate constants of the stabilizing reactions to zero, i.e., $k_{4}=k_{5}=k_{6}=0$. The figure shows the (quadratic) increase of $Z$ with time. The inset shows the constant $X$ level and the linear increase in $Y$. 
protein $Y$. This protein catalyses a reaction leading to the end product $Z$ that inhibits further synthesis of this mRNA species [Fig. 1(a)].

\section{EXPLANATION OF OSCILLATIONS}

The "driving force" of the oscillations is due to the amplification reactions $\mathrm{R} 2$ and $\mathrm{R} 3$. The amplification can be recognized by setting $k_{4}=k_{5}=k_{6}=0$. When the synthesis of $X$ is inhibited $\mathrm{d} X / \mathrm{d} t \rightarrow 0$, i.e., $X$ becomes constant and attains a steady-state value $X_{\mathrm{SS}}$. Subsequently, the rate $\mathrm{d} Y / \mathrm{d} t=k_{2} X_{\mathrm{SS}}$ will also be constant and will lead to a linear increase of $Y$. Subsequent integration of eqn (3) $\left(k_{6}=0\right)$ shows that $Z$ increases quadratically with time [Fig. 1(b)].

If reactions R4, R5, and R6 are operative at higher values of $Z$, the steady-state value $X_{\mathrm{SS}}$ will eventually decrease because of reaction R4. When $X$ decreases, the synthesis rate of $Y$ will also decrease (because $k_{2} X$ decreases). In addition, the concentration of $Y$ will decrease because of reaction R5. This will finally lead to a decrease in $\mathrm{d} Z / \mathrm{d} t$ and in the level of $Z$ such that the inhibition of the formation of $X$ will disappear. $X$ will be produced and the cycle restarted.

According to Franck $(1978,1989)$ the driving forces in oscillatory reactions are based on self-amplifying processes. Because amplification drives the system away from its steady state (destabilizes the system), Franck noticed that in order to obtain oscillations additional stabilizing reactions must oppose the amplification. This simultaneous action of stabilizing and destabilizing reactions has been termed antagonistic feedback (Franck, 1978, 1989), and is a necessary (but not sufficient) condition for oscillations. In the Goodwin oscillator, R4, R5, and R6 [Fig. 1(a)] are such stabilizing processes.

The existence of antagonistic feedback is not a sufficient condition for oscillations. Depending on the values chosen for the rate constants, we may observe limit cycle oscillations, damped oscillations or a monotonic non-oscillatory approach to a steady state. Besides numerical computations, linear stability analysis (Murray, 1989) has been used to assure whether a certain set of rate constant values result in a stable or in an unstable steady state (damped or limit cycle oscillations).

The exponent in the $Z^{9}$ term in eqn (1) is known to be unrealistically high. In order to decrease this exponent, the number of amplifying reactions can be increased (Rensing \& Schill, 1985). However, in the present study we wish to keep the number of parameters and rate constants at a minimum and therefore study only the dynamics of eqns (1-3).
With the rate constant values chosen in our computations, "soft" almost sinusoidal oscillations are observed. "Soft" means that the system needs a considerable time and many transient cycles to reach the final state (limit cycle or stable steady state) after a perturbation.

\section{The Temperature-Compensated Goodwin Model}

Temperature is introduced into the model by the Arrhenius equation, which links rate constant $k_{\mathrm{i}}$ to temperature $T$ :

$$
k_{\mathrm{i}}=A_{\mathrm{i}} \exp \left\{-\frac{E_{\mathrm{i}}}{R T}\right\}
$$

in which the pre-exponential factor $A_{\mathrm{i}}$ is treated as a constant and $E_{\mathrm{i}}$ is the activation energy of reaction $R i$. Both $A_{\mathrm{i}}$ and $E_{\mathrm{i}}$ are temperature-independent. $283 \mathrm{~K}$ or $298 \mathrm{~K}$ are used as reference temperatures, which means that the selected values of $k_{1}-k_{6}$ refer to one of these temperatures. The choice of activation energies $E_{\mathrm{i}}$ allows determination of the $A_{\mathrm{i}}$ 's. Equation (4) can then be used to calculate the $k_{\mathrm{i}}$ 's at different temperatures $T$. In biological terms, reactions R1-R6 will be enzymatic and involve more temperaturedependent [eqn (4)] rate constants (Laidler \& Peterman, 1979). The use of a single rate constant $k_{\mathrm{i}}$ for process $R i$ as chosen in this work represents an approximation.

For most chemical oscillator models it is impossible to describe the influence of temperature on period length analytically. Therefore, a numerical solution must be sought. An approximate analytical expression, however, can be obtained by writing the period length $P$ as a power law function of the rate constants (Ruoff, 1992, 1994, 1995). In case of the Goodwin model the expression takes the form:

$$
P_{\text {appr }}=\tau_{0} \prod_{n=1}^{6}\left(k_{\mathrm{i}}\right)^{\alpha_{\mathrm{i}}}
$$

where $\alpha_{i}$ are empirical (but otherwise constant) parameters which can be found by calculating $\partial \log P_{\text {num }} / \partial \log k_{\mathrm{i}}$ from numerically determined period lengths, $P_{\text {num }} \cdot \tau_{0}$ is a constant that is dependent on the reference rate constants. We have compared $P_{\text {appr }}$ with $P_{\text {num }}$ for a variety of rate constant values and found that eqn (5) describes quite well the period of the oscillations as a function of $k_{\mathrm{i}}$.

The condition for temperature-compensation is given by

$$
\frac{\partial P_{\text {appr }}}{\partial T}=0
$$


TABLE 1

Period $\mathrm{P}$ as a function of temperature for antagonistically balanced and non-balanced $\mathrm{E}_{i}$ combinations*

\begin{tabular}{|c|c|c|c|c|c|c|c|c|c|c|}
\hline $\begin{array}{l}E_{1} \\
(\mathrm{~J} / \mathrm{mol})\end{array}$ & $\begin{array}{c}E_{2} \\
(\mathrm{~J} / \mathrm{mol})\end{array}$ & $\begin{array}{c}E_{3} \\
(\mathrm{~J} / \mathrm{mol})\end{array}$ & $\begin{array}{c}E_{4} \\
(\mathrm{~J} / \mathrm{mol})\end{array}$ & $\begin{array}{c}E_{5} \\
(\mathrm{~J} / \mathrm{mol})\end{array}$ & $\begin{array}{c}E_{6} \\
(\mathrm{~J} / \mathrm{mol})\end{array}$ & $P\left(15^{\circ} \mathrm{C}\right)$ & $P\left(20^{\circ} \mathrm{C}\right)$ & $P\left(25^{\circ} \mathrm{C}\right)$ & $P\left(30^{\circ} \mathrm{C}\right)$ & $P\left(35^{\circ} \mathrm{C}\right)$ \\
\hline $3.96 \times 10^{4}$ & $1.34 \times 10^{5}$ & $9.11 \times 10^{4}$ & $2.21 \times 10^{2}$ & $5.93 \times 10^{2}$ & $9.28 \times 10^{2}$ & 24.1 & 23.1 & 23.1 & 23.0 & 22.9 \\
\hline $7.66 \times 10^{4}$ & $4.66 \times 10^{4}$ & $1.18 \times 10^{5}$ & $3.44 \times 10^{2}$ & $3.39 \times 10^{2}$ & $9.08 \times 10^{2}$ & 23.7 & 23.1 & 23.0 & 22.7 & 22.8 \\
\hline $2.11 \times 10^{5}$ & $6.49 \times 10^{3}$ & $6.11 \times 10^{4}$ & $7.54 \times 10^{2}$ & $6.60 \times 10^{2}$ & $3.97 \times 10^{2}$ & 24.4 & 23.0 & 22.5 & 22.9 & 22.1 \\
\hline $2.36 \times 10^{4}$ & $9.26 \times 10^{4}$ & $6.25 \times 10^{4}$ & $7.63 \times 10^{2}$ & $1.92 \times 10^{2}$ & $2.02 \times 10^{2}$ & 23.1 & 23.1 & 22.9 & 22.5 & 22.8 \\
\hline $2.36 \times 10^{4}$ & $9.26 \times 10^{4}$ & $6.25 \times 10^{4}$ & $7.63 \times 10^{2}$ & $1.00 \times 10^{5}$ & $2.02 \times 10^{2}$ & 66.7 & 48.3 & 36.7 & 29.0 & 23.5 \\
\hline $2.09 \times 10^{4}$ & $1.68 \times 10^{4}$ & $2.42 \times 10^{4}$ & $5.96 \times 10^{4}$ & $9.67 \times 10^{3}$ & $2.79 \times 10^{4}$ & 37.6 & 28.4 & 22.5 & 18.1 & 14.9 \\
\hline $2.38 \times 10^{4}$ & $5.69 \times 10^{4}$ & $1.63 \times 10^{4}$ & $1.88 \times 10^{4}$ & $4.77 \times 10^{4}$ & $3.41 \times 10^{4}$ & 38.3 & 28.7 & 22.6 & 18.1 & 14.5 \\
\hline $3.68 \times 10^{4}$ & $4.64 \times 10^{4}$ & $4.16 \times 10^{4}$ & $1.16 \times 10^{4}$ & $2.01 \times 10^{4}$ & $2.75 \times 10^{3}$ & 27.5 & 25.1 & 22.8 & 20.7 & 19.3 \\
\hline $3.11 \times 10^{4}$ & $4.55 \times 10^{3}$ & $2.58 \times 10^{4}$ & $3.72 \times 10^{3}$ & $5.01 \times 10^{4}$ & $1.09 \times 10^{4}$ & 32.0 & 26.3 & 22.6 & 19.5 & 17.2 \\
\hline $5.48 \times 10^{4}$ & $6.83 \times 10^{3}$ & $1.99 \times 10^{4}$ & $5.12 \times 10^{4}$ & $3.76 \times 10^{4}$ & $2.14 \times 10^{3}$ & 38.1 & 28.9 & 22.7 & 18.4 & 15.3 \\
\hline $3.49 \times 10^{4}$ & $3.20 \times 10^{2}$ & $3.47 \times 10^{4}$ & 0 & 0 & 0 & 22.9 & 22.3 & 22.3 & 22.6 & 22.6 \\
\hline $1.76 \times 10^{4}$ & $2.00 \times 10^{4}$ & $4.05 \times 10^{4}$ & 0 & 0 & 0 & 22.8 & 22.6 & 22.5 & 22.6 & 22.6 \\
\hline $2.53 \times 10^{4}$ & $5.15 \times 10^{4}$ & $7.39 \times 10^{3}$ & 0 & 0 & 0 & 22.9 & 22.4 & 22.4 & 22.6 & 22.5 \\
\hline $5.17 \times 10^{4}$ & $2.36 \times 10^{4}$ & $3.16 \times 10^{4}$ & 0 & 0 & 0 & 22.9 & 22.5 & 22.5 & 22.6 & 22.6 \\
\hline $4.12 \times 10^{4}$ & $3.39 \times 10^{4}$ & $1.73 \times 10^{4}$ & 0 & 0 & 0 & 23.0 & 22.4 & 22.4 & 22.6 & 22.6 \\
\hline
\end{tabular}

$* k_{1}=k_{2}=k_{3}=1.0 ; k_{4}=k_{5}=0.2 ; k_{6}=0.1 ; T_{\text {ref }}=298 \mathrm{~K}$. Rows $1-4:$ activation energies $E_{4}-E_{6}$ were randomly selected in the range $0-1000 \mathrm{~J} / \mathrm{mol}$; activation energies $E_{2}$ and $E_{3}$ were randomly selected in the range $0-1.5 \times 10^{5} \mathrm{~J} / \mathrm{mol} ; E_{1}$ was calculated according to eqn (10). Row 5: first period of damped oscillations when rate constant $k_{5}$ is decreased to $0.05\left(T_{\text {ref }}=298 \mathrm{~K}\right)$ and $E_{5}$ increased to $1 \times 10^{5} \mathrm{~J} / \mathrm{mol}$. Rows 6-10: all activation energies were randomly selected in the range $0-6.0 \times 10^{4} \mathrm{~J} / \mathrm{mol}$. Rows $11-15$ : activation energies $E_{1}-E_{3}$ were randomly selected in the range $0-6.0 \times 10^{4} \mathrm{~J} / \mathrm{mol}$.

When inserting the Arrhenius eqn (4) into eqn (5) and making the derivation with respect to temperature, one obtains:

$$
\frac{\partial P_{\text {appr }}}{\partial T}=\frac{1}{R T^{2}}\left[\sum_{i=1}^{6} \alpha_{\mathrm{i}} E_{\mathrm{i}}\right] P_{\mathrm{appr}}
$$

The condition for temperature-compensation [eqn (6)] implies that:

$$
\sum_{n=1}^{6} \alpha_{\mathrm{i}} E_{\mathrm{i}}=0
$$

In other words: whenever the $\alpha_{i}$-weighted sum of the activation energies is zero (or approximately zero) the oscillator is temperature-compensated. The reason why eqn (8) can be zero is due to the fact that all component processes in the oscillator can be divided into period-increasing $\left(\mathrm{P}^{+}\right)$and period-decreasing $\left(\mathrm{P}^{-}\right)$reactions. $\mathrm{P}^{+}$reactions will have positive $\alpha_{i}$ values, while $\mathrm{P}^{-}$reactions will have negative $\alpha_{i}$ values. Because the $\mathrm{P}^{+}$and $\mathrm{P}^{-}$reactions are associated with the destabilizing (amplifying) and stabilizing reactions of the oscillator, we have termed eqn (8) as antagonistic balance in temperature (Ruoff, 1994).

In case eqn (8) is not fulfilled the period will be temperature-dependent [eqn (5)]:

$$
P_{\text {appr }}=C \exp \left\{-\left[\sum_{i=1}^{6} \alpha_{\mathrm{i}} E_{\mathrm{i}}\right] / R T\right\}
$$

where $\mathrm{C}$ is a (temperature-independent) constant. Experimental results show that chemical oscillatory reactions follow the exponential temperature dependence of the period [eqn (9)] often with high precision (Ruoff, 1995).

For the Goodwin oscillator the $\alpha_{\mathrm{i}}$ values of the stabilizing reactions $\mathrm{R} 4, \mathrm{R} 5, \mathrm{R} 6$ are negative, while the $\alpha_{i}$ values of the amplifying R1, R2, R3 are slightly positive, and eqn (8) can be written as:

$$
0.0023\left(E_{1}+E_{2}+E_{3}\right)=0.3583\left(E_{4}+E_{5}\right)+0.3416 E_{6}
$$

The first four rows in Table 1 show that for $E_{\mathrm{i}}$-combinations that follow eqn (10), small changes in the period length are observed as the temperature is varied. On the other hand, when all six $E_{\mathrm{i}}$ values are allowed to change randomly (rows 6-10), and eqn (10) is not obeyed, a strong temperature dependence appears.

Equation (10) implies that the average activation energies $E_{1}, E_{2}, E_{3}$ should be about 150 times larger than the average value of $E_{4}, E_{5}$, or $E_{6}$. If the average, $E_{1}, E_{2}, E_{3}$ value is about $50 \mathrm{~kJ} / \mathrm{mol}$ (that would result in rate constants with $\mathrm{Q}_{10}$ values of 2), then $E_{4}, E_{5}$ and $E_{6}$ should be near $0 \mathrm{~kJ} / \mathrm{mol}$ to obtain temperaturecompensation. This precise situation is shown in Table 1, rows 11-15.

Row 5 in Table 1 illustrates the effect when the rate constant of the degradation of protein $Y$ (process R5) is decreased. In this case temperature-compensation is 
TABLE 2

Period $\mathrm{P}$ as a function of temperature for damped and limit cycle oscillations ${ }^{a}$

\begin{tabular}{|c|c|c|}
\hline Temperature (K) & Period & Stability of steady state \\
\hline 273 & 23.7 & stable $^{b}$ \\
\hline 275 & 23.3 & stable $^{b}$ \\
\hline 277 & 22.8 & stable \\
\hline 279 & 22.7 & stable $^{\mathrm{b}}$ \\
\hline 281 & 22.7 & stable $^{\mathrm{b}}$ \\
\hline 283 & 22.7 & stable $^{\mathrm{b}}$ \\
\hline 285 & 22.7 & stableb \\
\hline 287 & 22.7 & stable ${ }^{\mathrm{b}}$ \\
\hline 289 & 22.7 & stable \\
\hline 291 & 22.7 & stable $^{\mathrm{b}}$ \\
\hline 293 & 22.7 & unstable $^{c}$ \\
\hline 295 & 22.7 & unstable \\
\hline 297 & 22.7 & unstable $^{\mathrm{c}}$ \\
\hline - & - & - \\
\hline 309 & 22.7 & unstable $^{\mathrm{c}}$ \\
\hline 311 & 22.7 & unstable $^{\mathrm{c}}$ \\
\hline 313 & 22.7 & unstable $^{c}$ \\
\hline \multicolumn{3}{|c|}{ 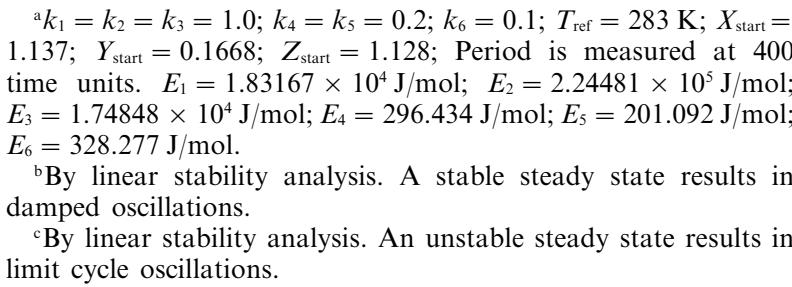 } \\
\hline
\end{tabular}

lost and the period length of the oscillator is altered (lengthened).

\section{Temperature-Entrainment of the Temperature- Compensated Model}

A temperature-compensated oscillator does not mean that the oscillations are independent of temperature. Here we show that the temperaturecompensated Goodwin model can be entrained by sinusoidal temperature cycles with rather small amplitudes.

\section{LIMIT-CYCLE OSCILLATOR CASE}

We consider a temperature-compensated limitcycle Goodwin oscillator having the following rate constant values at $283 \mathrm{~K}: k_{1}=1.0 ; \quad k_{2}=1.0$; $k_{3}=1.0 ; k_{4}=0.1 ; k_{5}=0.1 ; k_{6}=0.1$. The activation energies for $k_{1}-k_{6}$ are: $E_{1}=1.83167 \times 10^{4} \mathrm{~J} / \mathrm{mol}$; $E_{2}=2.24481 \times 10^{5} \mathrm{~J} / \mathrm{mol} ; \quad E_{3}=1.74848 \times 10^{4} \mathrm{~J} / \mathrm{mol} ;$ $E_{4}=296.434 \mathrm{~J} / \mathrm{mol} ; \quad E_{5}=201.092 \mathrm{~J} / \mathrm{mol} ; \quad E_{6}=$ $328.277 \mathrm{~J} / \mathrm{mol}$. In the studied temperature range from $273 \mathrm{~K}$ to $313 \mathrm{~K}$ the period of the unperturbed oscillator, $P_{\text {c(ircadian) }}$, changes from 36.8 to 37.6 with a maximum of 38.0 at $283 \mathrm{~K}$.

When the period of the temperature cycle, $P_{\text {temperature) }}$, is shorter than $P_{\mathrm{c}}$, entrainment is observed until $P_{\mathrm{t}}$ reaches about 30 [Fig. 2(A)]. For $P_{\mathrm{t}}$ lower than 30, entrainment is not observed and the oscillator resets to its original period of 38 [Fig. 2(b)]. An increase of the temperature amplitude at low $P_{\mathrm{t}}$ does not enforce entrainment, but leads to a irregular/chaotic response.

When $P_{\mathrm{t}}$ is longer than $P_{\mathrm{c}}$ (38.0) temperatureentrainment can be observed even at rather long $P_{\mathrm{t}}$ values (data not shown). When even longer temperature cycles are applied, one observes oscillations with period $P_{\mathrm{c}}$ superimposed on the entrained (enforced) rhythm. It is also observed that when the amplitude of the entraining temperature rhythm increases, the amplitude of the superimposed oscillations decrease.

TEMPERATURE-COMPENSATION IN DAMPED SYSTEMS AND TEMPERATURE EFFECTS ON NONOSCILLATORY STEADY STATES

Temperature-compensation may also be observed in damped oscillations, i.e., when the Goodwin model approaches a stable steady state (Table 2). From these results we conclude that antagonistic balance applies not only to pure limit cycle oscillations, but apparently also to stable and unstable oscillations!

However non-oscillatory (damped stable steady) states can also be influenced by temperature. Short $P_{t}$ values in damped systems lead to irregular oscillations, which is similar to the situation when limit cycle oscillations are perturbed.

\section{LOSS OF OSCILLATION STABILITY AT LOW TEMPERATURE}

Table 2 illustrates another behavior often observed experimentally: when the temperature is lowered the oscillations of the Goodwin model become damped. In the particular case studied here, the steadystate changes its stability between $291 \mathrm{~K}$ and 293 K. Interestingly, the oscillator's temperaturecompensation is independent of the stability of the steady state.

\section{Phase Resetting by Temperature Steps}

When the temperature is changed from $273 \mathrm{~K}$ to $278 \mathrm{~K}$ for the temperature-compensated limit cycle oscillator $\left(k_{1}=1.0, \quad k_{2}=1.0, \quad k_{3}=1.0, \quad k_{4}=0.1\right.$, $k_{5}=0.1, k_{6}=0.1, T_{\text {ref }}=283 \mathrm{~K}, E_{1}=1.83167 \times 10^{4} \mathrm{~J} /$ $\mathrm{mol} ; E_{2}=2.24481 \times 10^{5} \mathrm{~J} / \mathrm{mol} ; E_{3}=1.74848 \times 10^{4} \mathrm{~J} /$ $\mathrm{mol} ; \quad E_{4}=296.434 \mathrm{~J} / \mathrm{mol} ; \quad E_{5}=201.092 \mathrm{~J} / \mathrm{mol} ;$ $E_{6}=328.277 \mathrm{~J} / \mathrm{mol}$ ) the oscillator approaches a new oscillatory state (limit cycle). Let us assume that the temperature step has been applied at time $t_{\text {per }}$ ("Phase of Perturbation"). We now compare the successive maxima in $X, Y$, or $Z$ for the unperturbed and perturbed oscillation when $t>t_{\text {per }}$. Let $T_{1}^{\mathrm{u}}, T_{2}^{\mathrm{u}}$, 

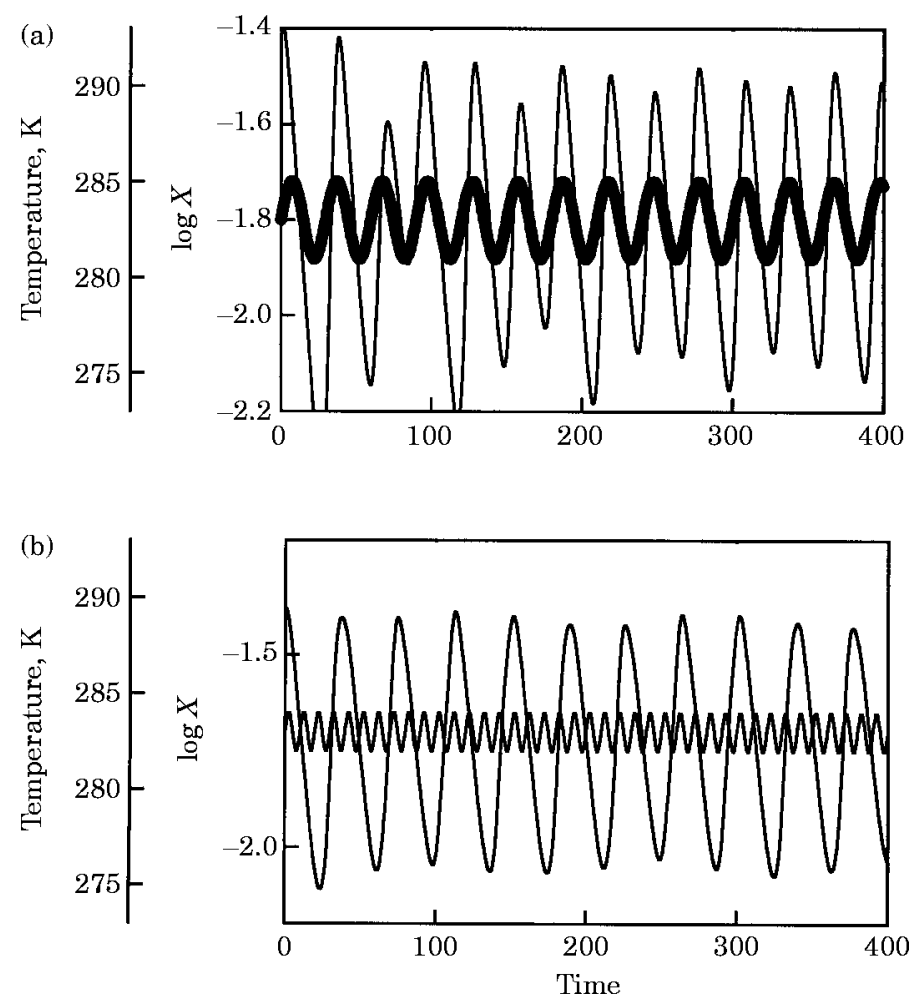

Fig. 2. (a) Temperature-entrainment using a sinusoidal temperature perturbation with $P_{\mathrm{t}}=30$ and a temperature amplitude of $1{ }^{\circ} \mathrm{C}$. The inner oscillation is the perturbing cycling temperature, while the outer oscillation is the responding $\log X$ value. (b) When $P_{\mathrm{t}}$ is decreased to 10 , the oscillator is no longer able to follow the perturbing rhythm and resets to its original unperturbed period $P_{\mathrm{c}}=38$.

$T_{3}^{\mathrm{u}}, \ldots, T_{\mathrm{n}}^{\mathrm{u}}, \ldots$ denote the series of successive $(X, Y$, or $Z$ ) maxima for the unperturbed oscillator when $t>t_{\mathrm{per}} . T_{1}^{\mathrm{p}}, T_{2}^{\mathrm{p}}, T_{3}^{\mathrm{p}}, \ldots, T_{\mathrm{n}}^{\mathrm{p}}, \ldots$ is the corresponding series for the perturbed oscillator. The phase shift $\Delta \Phi_{\mathrm{n}}$ related to the $\mathrm{n}^{\text {th }}(X, Y$, or $Z)$ maximum is defined as

$$
\Delta \Phi_{\mathrm{n}}=T_{\mathrm{n}}^{\mathrm{u}}-T_{\mathrm{n}}^{\mathrm{p}}
$$

A phase response curve is a relationship that shows any phase shift $\Delta \Phi$ as a function of $t_{\text {per }}$. Negative phase shifts correspond to phase delays, while positive phase shifts correspond to phase advances.

THE PROBLEM OF ASSIGNING A UNIQUE PHASE SHIFT FOR TEMPERATURE STEPS

Figure 3 shows the calculated $\Delta \Phi_{\mathrm{n}}$ values as a function of $n$ for a $273 \mathrm{~K} \rightarrow 278 \mathrm{~K}$ step. After an initial fall, $\Delta \Phi_{\mathrm{n}}$ values decrease linearly with $n$. The reason for this linear decrease in $n$ lies in the fact that despite the oscillator's temperature-compensation the period at $278 \mathrm{~K}$ is slightly longer than the period at $273 \mathrm{~K}$. From Fig. 3 it is apparent that there is a problem in assigning a unique $\Delta \Phi$ among the $\Delta \Phi_{\mathrm{n}}$ values that would represent 'the' phase shift. To solve this problem we have made two linear extrapolations from the initial and final $\Delta \Delta \Phi_{\mathrm{n}} / \Delta n$ values and estimate $\Delta \Phi$ as that $\Delta \Phi_{\mathrm{n}}$ value which corresponds to the intersection of the two lines (Fig. 3).

The variation of $\Delta \Phi_{\mathrm{n}}$ with $n$ in Fig. 3 is one of six possibilities that can occur when studying phase shifts

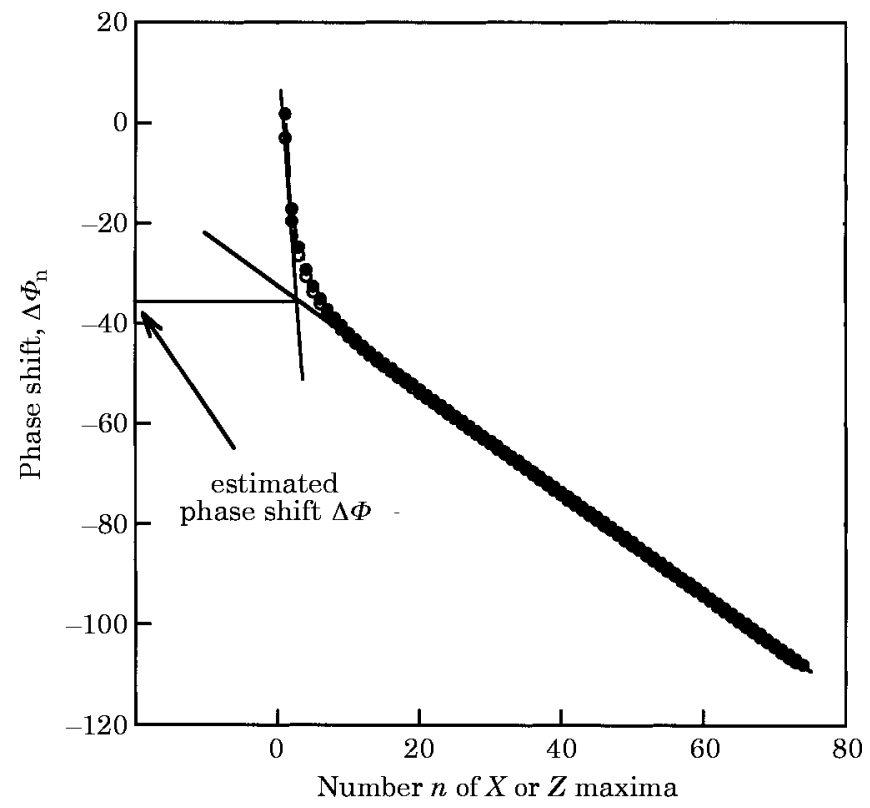

FIG. 3. Phase shifts $\Delta \Phi_{\mathrm{n}}$ as a function of $n$ for $X(\bullet)$ maxima. The estimated phase shift $\Delta \Phi$ is indicated. 

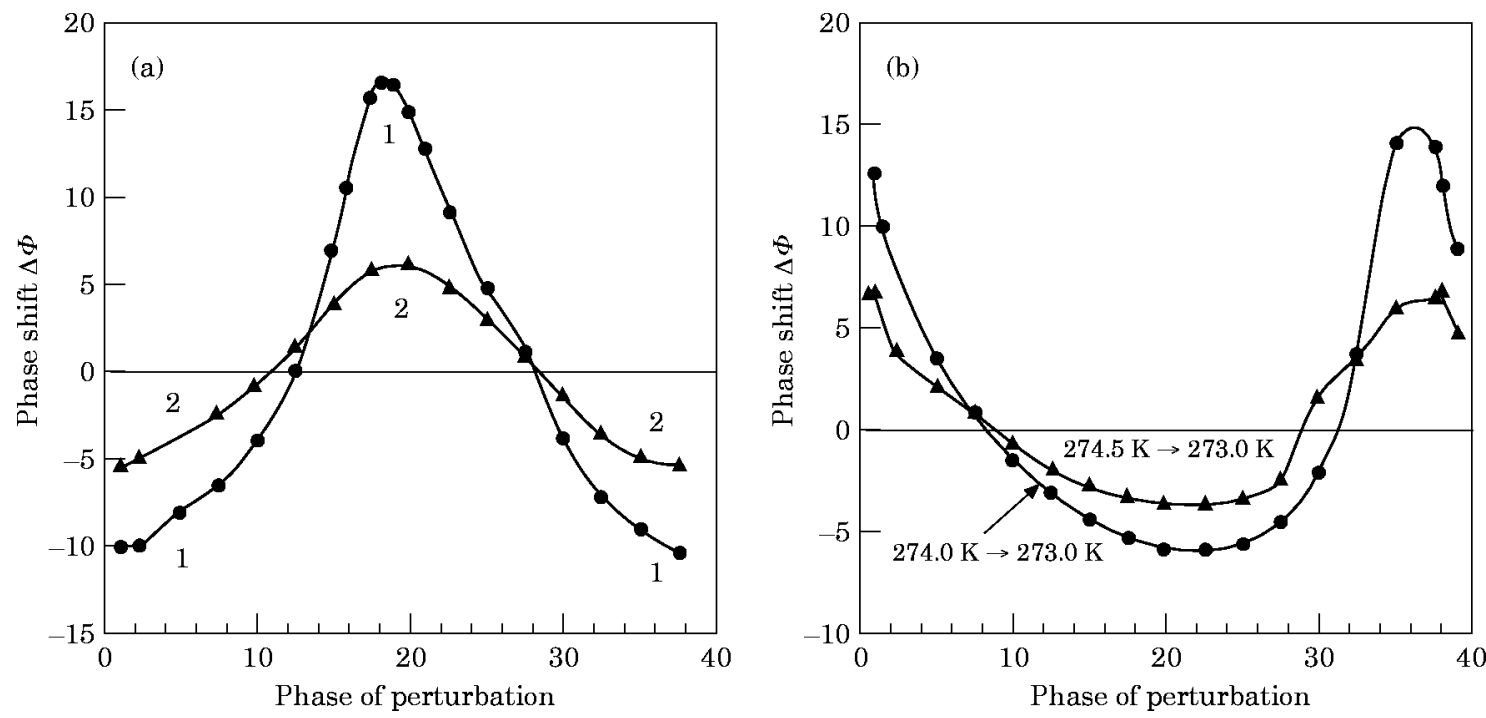

Fig. 4. (a) Estimated phase shifts $\Delta \Phi$ for temperature step-ups. $1: 273 \mathrm{~K} \rightarrow 274 \mathrm{~K} ; 2: 273.0 \mathrm{~K} \rightarrow 273.5 \mathrm{~K}$. (b) Estimated phase shifts $\Delta \Phi$ for temperature step-downs. Zero corresponds to the $X$ maximum.

due to temperature steps. Three possibilities occur because the final frequency of the perturbed oscillator may be (i) the same, (ii) lower, or (iii) higher than the initial frequency. For each of these three possibilities there are two additional possibilities, because the initial change of $\Delta \Phi_{\mathrm{n}}$ may either increase or decrease.

Figure 4 shows phase shifts determined for temperature step-ups and temperature step-downs. It may be noted, that for an increasing and decreasing temperature step positive and negative phase shifts are observed.

\section{Phase Resetting by Temperature Pulses}

PRCs derived from pulsed perturbations of the circadian oscillator are most common in the experimental literature but have also been studied thoroughly from the theoretical point of view (Winfree, 1980). The effect of a temperature pulse on phase resetting is more straightforward to analyse than the effect of a temperature step: after the applied pulse the oscillator returns to its initial oscillatory state (with exactly the same period) and reaches a constant phase difference between unperturbed and perturbed oscillator. The definition of phase shifts $\Delta \Phi_{\mathrm{n}}$ is that of eqn (9). The final phase shift $\Delta \Phi$ is the limiting phase shift for large $n$, i.e.,

$$
\Delta \Phi=\lim _{\mathrm{n} \rightarrow \infty} \Delta \Phi_{\mathrm{n}} .
$$

In practice, it was sufficient to calculate $\Delta \Phi$ as the $\Delta \Phi_{\mathrm{n}}$ value when $\mathrm{n}=20$, i.e., after 800 time units after the pulse has been applied.

The main variables of the perturbing pulse are its length and height, which are both affecting the resulting degree of $\Delta \Phi$ (for review see Winfree, 1980). However, not only was a dose-dependence of $\Delta \Phi$ observed but also a shift of the position of the PRC with respect to the phase of the applied pulse (Taylor et al., 1982). It is, therefore, useful to determine maximal or minimal $\Delta \Phi$ independent of its phase position. When varying length and height of temperature pulses the resulting experimental dose response curves cannot always be identified to a linear or nonlinear relation (Cornelius \& Rensing, 1982) which would allow conclusions with respect to the temperature dependence of the involved processes.

In the Goodwin model we observed the following dependencies. Figure 5(a) shows the phase response curves obtained for $273 \mathrm{~K} \rightarrow 275 \mathrm{~K}$ temperature pulses with variable pulse lengths. Figure 5(b) shows that both the maximal advance and delay phase shifts are linearly dependent on pulse length. Figure 6 shows the behavior of the phase response curve at constant pulse length with varying pulse height $\Delta T$. In contrast to the pulse length, the maximal phase shifts are now exponential functions of the temperature.

\section{Discussion}

The Goodwin model [Fig. 1(a)] is a simple oscillatory system containing an inhibitory feedback. Such a feedback system has now been recognized as part of the circadian oscillator of Drosophila melanogaster and in Neurospora crassa (Hardin et al., 1992; Aronson et al., 1994; Zeng et al., 1994).

Our results show that the modified Goodwin model is in qualitative agreement with the main features 

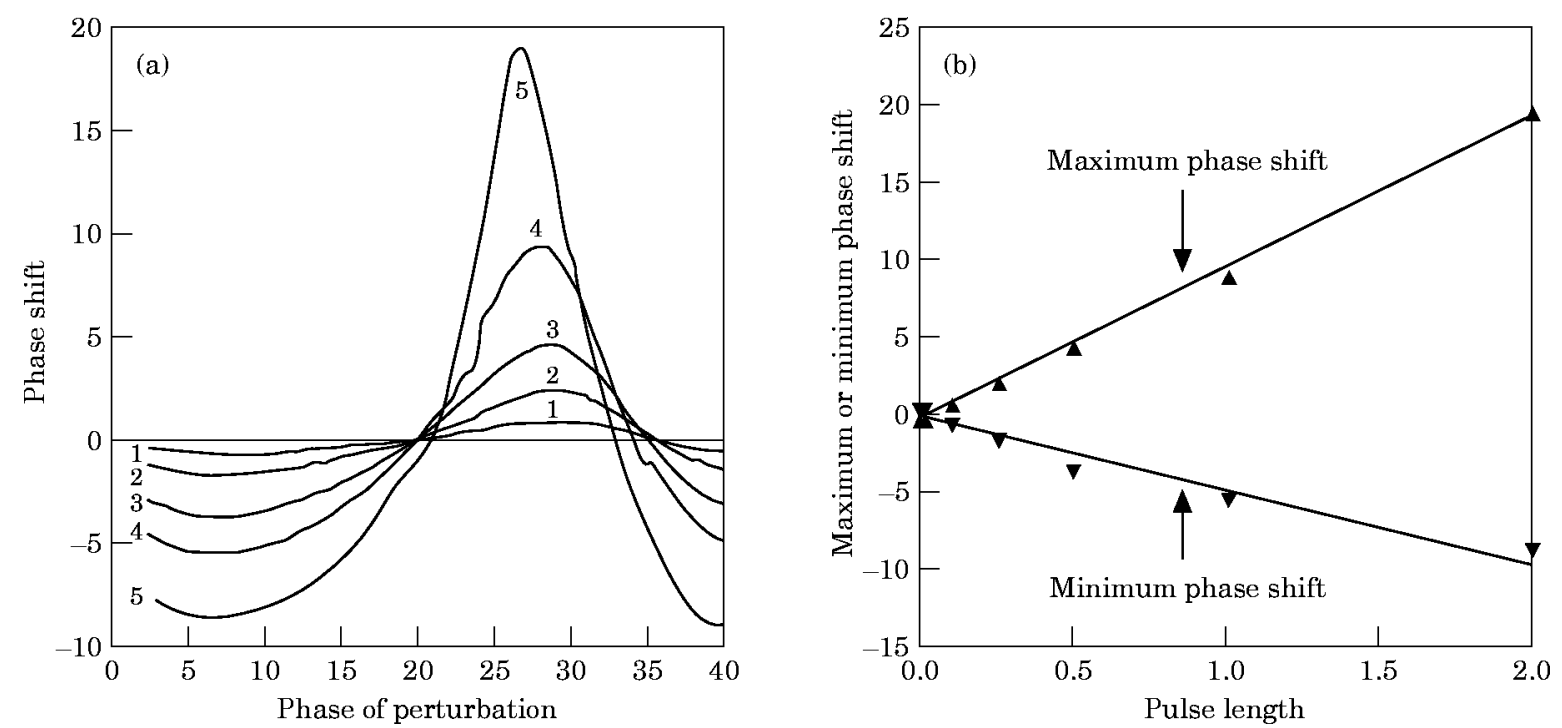

Fig. 5. (a) Phase response curve for $273 \mathrm{~K} \rightarrow 275 \mathrm{~K}$ temperature pulses with variable pulse length. Zero phase corresponds to the $X$ maximum. Pulse lengths: $1,0.1 ; 2,0.25 ; 3,0.5 ; 4,1.0 ; 5,2.0$. (b) Maximal advance and delay phase shift show a linear dependence on pulse lengths.

of the circadian oscillator observed experimentally when studying temperature-entrainment and phase resetting.

\section{ACTIVATION ENERGY REQUIREMENTS FOR TEMPERATURE- COMPENSATION}

Antagonistic balance [eqn (8)] implies temperaturecompensation for different but constant temperature levels. The loss of temperature compensation by (point) mutations in organisms like Neurospora (Lakin-Thomas et al., 1990; Aronson et al., 1994) could be interpreted in terms of antagonistic balance

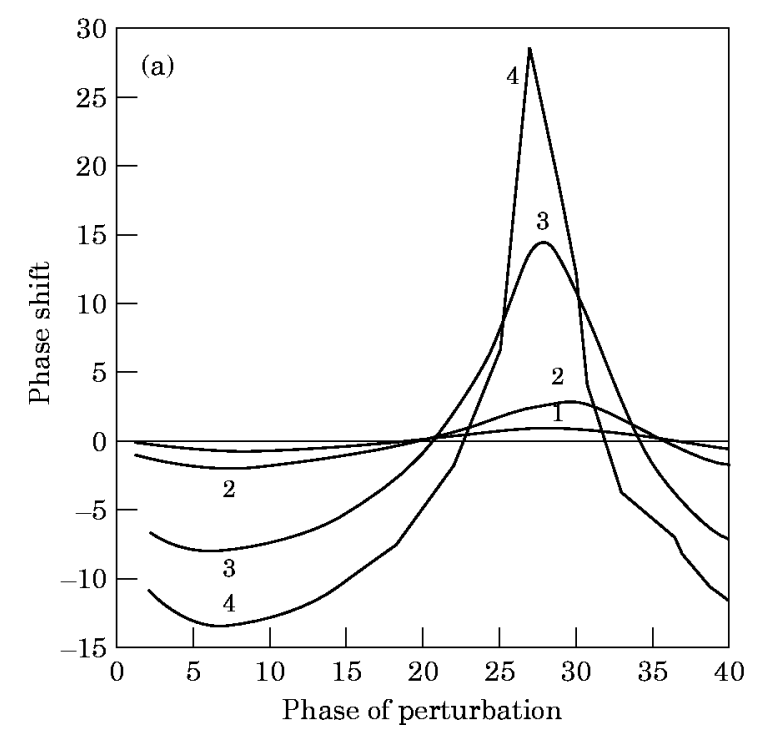

in the following way: the point mutation alters the degradation rate of a protein that catalyses one of the reactions that participate in the oscillatory mechanism. This causes a change in activation energy and the reaction rate catalysed by the enzyme. A large change in activation energy and reaction rate then destroys the antagonistic balance and leads to a different period length as well as abolishment of temperaturecompensation or even of oscillations (Table 1, row 5; Ruoff, 1994; Aronson et al., 1994). Equation (8) also implies that whenever the activation energies $E_{4}, E_{5}$, and $E_{6}$ of the degradation reactions are low, the

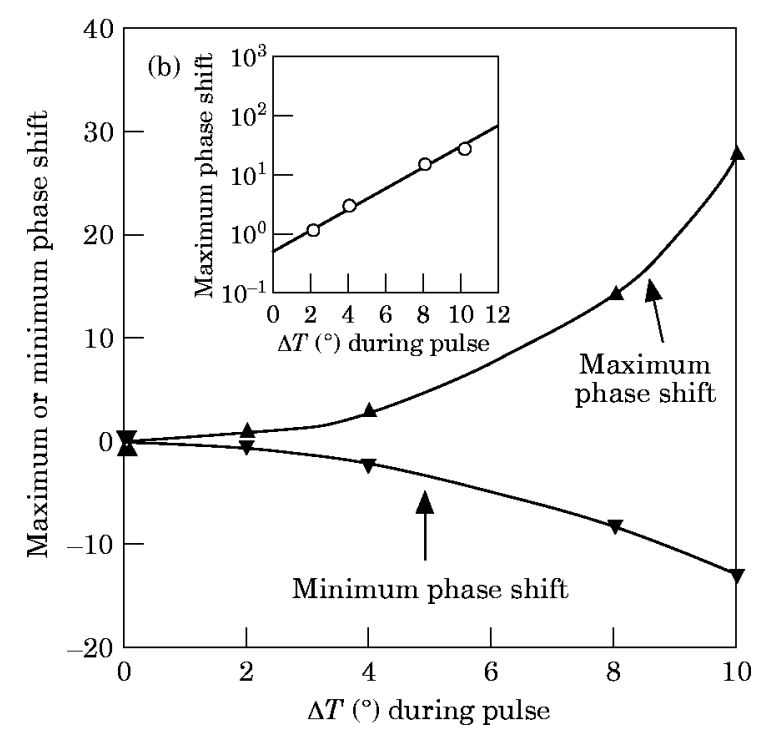

FIG. 6. (a) Effect of temperature pulse height $\Delta T$ on phase response curves for temperature pulses with 0.1 time units pulse length. Initial temperature is $273 \mathrm{~K}$. Pulse heights: 1: $2 \mathrm{~K} ; 2: 4 \mathrm{~K} ; 3: 8 \mathrm{~K}$; 4: $10 \mathrm{~K}$. (b) Maximal advance and delay phase shifts show an exponential dependence on pulse heights $\Delta T$. Zero phase corresponds to the $X$ maximum. 
activation energies $E_{1}, E_{2}$, and $E_{3}$ can be varied in a relatively large range without affecting temperaturecompensation (Table 1). It is an interesting question whether this particular way to achieve temperaturecompensation is of physiological significance. Mechanisms compensating for the effects of different temperatures, commonly called temperature adaptation, are by no means restricted to circadian rhythms. Protein synthesis and degradation, in particular, are balanced, i.e., moderate temperature changes influence both processes in the same direction, thus leaving the protein level relatively constant after a short transient change (DeMaio et al., 1993; Mohsenzadeh et al., 1994; Rensing et al., 1995). In addition, each of the two processes is apparently adapting, involving protein phosphorylation changes of the initiation or elongation factors (Duncan \& Hershey, 1989) and de novo synthesis of proteinases (Mohsenzadeh et al., 1994). The fluidity of membranes is another property of cells which is often adapting to different temperatures, e.g., kept rather constant, a phenomenon called homeoviscous adaptation (Thompson, 1980).

\section{DAMPED TEMPERATURE-COMPENSATED OSCILLATIONS}

One of the interesting features of the modified Goodwin Oscillator is that even damped oscillations show temperature-compensation. It may be noted that the transition between damped and limit cycle oscillations occurs smoothly with no apparent changes in period (Table 2). In view of the condition of antagonistic balance, temperature-compensation in damped oscillatory responses should also be observed in other oscillators. This may lead to a slightly alternative view of "circadian responses" of organisms: while models for circadian organization mostly included an unspecified limit cycle "master clock", there may exist instead a set of entrainable structurally unstable physiological functions that may result in a (more or less damped but temperaturecompensated) oscillatory response (Sinha, 1981, 1983). The consideration of damped systems easily accounts for the fact that many circadian rhythms become damped once the organisms are under constant conditions [for example light (Lillo, 1984; Lillo \& Ruoff, 1984)]. The consideration of an entrainable and damped-oscillatory physiology is an alternative to the concept of one internal limit cycle master clock.

\section{ENTRAINMENT BY TEMPERATURE CYCLES}

Both temperature-compensated limit cycles and a (temperature-compensated) non-oscillatory steady state can readily be entrained or (the latter) forced even by a change in temperature of a few degrees centigrade. This is in agreement with experiments which have shown that circadian rhythms can be entrained by temperature cycles of only a few degrees centigrade amplitude (Oltmanns, 1960; Hoffmann, 1969a, b; Lindberg \& Hayden, 1974; Tokura \& Oishi, 1985).

However, when the perturbing temperature period becomes too short, the Goodwin oscillator is not able to keep up with the perturbing rhythm and oscillates with its "natural" frequency, a well-known phenomenon for circadian rhythms. Under these circumstances an increase in temperature is not able to enforce regular oscillations: the response becomes irregular.

\section{PHASE RESETTING BY TEMPERATURE STEPS AND PULSES}

The phase response curves of the Goodwin oscillator are qualitatively similar to temperature step perturbations of the circadian rhythm of eclosion in Drosophila pseudoobscura (Zimmerman et al., 1968) for small increasing temperature steps. However, studies on models based on autocatalytical amplification (Ruoff, unpublished) show the opposite behavior: temperature step-ups show delayed phase shifts with a minimum, while temperature step-downs show positive phase shifts with a maximum. These results indicate a major difference between the phase response behavior of oscillator models containing an autocatalytical reaction (like the Brusselator or the Kauffman-Wille model) and oscillators containing an inhibitory reaction as the Goodwin model.

A difference, however, between the data observed for Drosophila pseudoobscura (Zimmerman et al., 1968) and the calculations presented here is that although positive temperature steps show a bellshaped phase response curve in the model [similar to that observed by Zimmerman et al., (1968)] the model calculation shows both negative and positive phase shifts. The same is true for negative temperature steps: the form of the calculated phase response curve [Fig. 4(b)] is quite similar to the experimental PRC (Zimmerman et al., 1968), with the exception that in the Goodwin model both positive and negative phase shifts are observed. On the other hand, the data for Neurospora crassa by Francis \& Sargent (1979) correspond better with our computations.

The PRC of the Goodwin model also shows qualitative agreement with the corresponding experimental analyses of temperature pulses (Zimmerman et al., 1968; Engelmann et al., 1974; Francis \& Sargent, 1979; Rensing et al., 1987; Nakashima, 1987; Gooch et al., 1994; Goto et al., 1994) in contrast to computations performed on autocatalytical models. 
Although the maximal advance and delay phase shifts depend linearly on pulse length [Fig. 5(b)], the variation with respect to the height of the temperature pulse is exponential [Fig. 6(b)]. This exponential dependence appears to be due to the Arrhenius-type of dependence between the rate constants and temperature [eqn (4)], and which becomes apparent during the transitory process induced by the temperature perturbation. On the other hand, when the phase shift is studied as a function of the pulse height, it is seen that the relationship phase shift-pulse height is dependent on the phase of perturbation. This can be seen in Fig. 6(a) in which the maximum phase shift is changing its position as a function of the pulse height. The behavior here appears similar to experimental observations by Francis \& Sargent (1979), Nakashima (1987) or Taylor et al. (1982) in the case of PRCs to protein synthesis inhibitors. Our calculations suggest that in order to see a possible exponential dependence between phase shift and the height of the temperature pulse, $\Delta T$, the maximum (or minimum) phase shift $\Delta \Phi_{\max }$ (or $\Delta \Phi_{\min }$ ) should be plotted against $\Delta T$. Existing experimental data of $\Delta \Phi_{\max }$ and $\Delta \Phi_{\min }$ vs. $\Delta T$ (Rensing et al., 1987) show an apparent linear relationship, indicating that the actual temperature dependence of the oscillatory processes is not exponential. This question, therefore, needs further experimental and theoretical analysis.

We find it encouraging that the Goodwin oscillator shows a much better qualitative agreement with experimental phase response curves than oscillator models in which the amplification/instability is based on autocatalysis.

\section{Conclusion}

The calculations show that a temperature-compensated Goodwin model can qualitatively describe many dynamic features of temperature-perturbed circadian rhythms, including temperature entrainment by small temperature variations and the shape of phase response curves of temperature steps and temperature pulses. Limit cycle oscillations may become damped by decreasing the temperature, however both limit cycle and damped oscillations still show temperaturecompensation.

$\begin{array}{ll} & \text { Abbreviations } \\ \Delta \Phi: & \text { a phase shift. } \\ \Delta \Phi_{\max }, \Delta \Phi_{\min }: & \begin{array}{l}\text { maximal or minimal phase shift. } \\ \Delta \Phi_{\mathrm{n}}:\end{array} \\ \text { the phase shift between the } \mathrm{n}^{\text {th }} \text { maxima }\end{array}$

of unperturbed and perturbed oscillator [eqn (9)].

$E_{\mathrm{i}}$ : the activation energy of rate constant $k_{\mathrm{i}}$ [eqn (4)].

$k_{\mathrm{i}}$ : the rate constant of reaction $R i$ (Fig. 1).

$P_{\text {appr }}: \quad$ an approximate period length calculated by [eqn (5)].

$P_{\mathrm{c}}$ : the (circadian) period length of an unperturbed oscillator.

$P_{\text {num }}: \quad$ the numerically calculated period length by solving differential eqns (1-3).

PRC: $\quad$ phase response curve.

$P_{\mathrm{t}}: \quad$ the period length of a perturbing sinusoidal temperature rhythm.

$T: \quad$ the temperature in Kelvin.

$t: \quad$ time.

$t_{\text {per }}$ : the time at which the perturbation of an oscillator is started.

$T_{\text {ref }}$ the (reference) temperature in Kelvin at which a certain set of rate constant values $k_{\mathrm{i}}$ are defined.

We thank S. Brody, W. J. Hastings, J. Woolum for valuable comments, and $\mathrm{M}$. Vicker for critically reading the manuscript.

\section{REFERENCES}

Aronson, B. D., Johnson, K. A., Loros, J. J. \& Dunlap. J. C. (1994a). Negative feedback defining a circadian clock: Autoregulation of the clock gene frequency. Science 263, 1578-1584.

Aronson, B. D., Keith, A. J. \& Dunlap, J. C. (1994b). Circadian clock locus frequency: Protein encoded by a single open reading frame defines period length and temperature compensation. Proc. Nat. Acad. Sci. U.S.A. 91, 7683-7687.

BALZER, I. \& HARDELAND, R. (1988). Influence of temperature on biological rhythms. Int. J. Biometeorol. 32, 231-241.

Cornelius, G. \& Rensing, L. (1982). Can phase response curves of various treatments of circadian rhythms be explained by effects on protein synthesis and degradation? BioSystems 15, 35-47.

DeMaio, A., Beck, S. C. \& Buchmann, T. G. (1993). Induction of translational thermotolerance in liver of thermally stressed rats. Eur. J. Biochem. 218, 413-420.

Drescher, K., Cornelius, G. \& Rensing, L. (1982). Phase response curves obtained by perturbing different variables of a $24 \mathrm{~h}$ model oscillator based on translational control. J. theor. Biol. 94, 345-353.

Duncan, R. F. \& Hershey, J. W. B. (1989). Protein synthesis and protein phosphorylation during heat stress, recovery, and adaptation. J. Cell Biol. 109, 1467-1481.

Edmunds, L. N. (1988). Cellular and Molecular Bases of Biological Clocks, New York: Springer-Verlag.

Engelmann, W., Eger, I., Johnsson, A. \& Karlsson, H. G. (1974). Effect of temperature pulses on the petal rhythm of Kalanchoe: An experimental and theoretical Study. Int. J. Chronobiol. 2, 347-358.

Field, R. J. \& Noyes, R. M. (1974). Oscillations in chemical systems. IV. Limit cycle behavior in a model of a real chemical reaction. J. Chem. Phys. 60, 1877-1884.

Francis, C. D. \& Sargent, M. L. (1979). Effects of temperature 
perturbations on circadian conidiation in Neurospora. Plant Physiol. 64, 1000-1004.

Franck, U. F. (1978). Chemical oscillations. Angew. Chem. Int. Ed. Engl. 17, 1-15.

Franck, U. F. (1989). Periodische Strukturen und Vorgänge in gleichgewichtsfernen physikalisch-chemischen Systemen. Nova acta Leopoldina NF 265, 109-131.

Gooch, V. D., Wehseler, R. A. \& Gross, C. G. (1994). Temperature effects on the resetting of the phase of the Neurospora circadian rhythm. J. Biol. Rhythms 9, 83-94.

Goodwin, B. C. (1963). Temporal Organization in Cells. A Dynamic Theory of Cellular Control Processes, New York: Academic Press.

Goodwin, B. C. (1965). Oscillatory behavior in enzymatic control processes, In Advances in Enzyme Regulation, Vol. 3 (Weber, G., ed.) pp. 425-438. Oxford: Pergamon Press.

Goto, R., Kaue, R., Megumi, M. \& Nakashima, H. (1994). Effects of temperature on the circadian conidiation rhythm of temperature-sensitive mutants of Neurospora crassa. Plant Cell Physiol. 35, 613-618.

Hardin, P. E., Hall, J. C. \& Rosbash, M. (1992). Circadian oscillators in period gene mRNA levels are transcriptionally regulated. Proc. Nat. Acad. Sci. U.S.A. 89, 11711-11715.

Hastings, J. W. \& SweEney, B. M. (1957). On the mechanism of temperature independence in a biological clock, Proc. Nat. Acad. Sci. U.S.A. 43, 804-811.

Hoffmann, K. (1969a). Die relative Wirksamkeit von Zeitgebern. Oecologia 3, 184-206.

HoFfmann, K. (1969b). Zum Einfluß der Zeitgeberstärke auf die Phasenlage der synchronisierten circadianen Periodik. Z. Vergl. Physiol. 62, 93-110.

Kauffman, S. \& Wille, J. J. (1975). The mitotic oscillator in Physarum polycephalum. J. theor. Biol. 55, 47-93.

Lakin-Thomas, P. L., Coté, G. G. \& Brody, S. (1990). Circadian rhythms in Neurospora crassa: Biochemistry and genetics. Crit. Rev. Microbiol. 17, 365-416.

Laidler, K. J. \& Peterman, B. F. (1979). Temperature effects in enzyme kinetics. In Methods in Enzymology-Enzyme Kinetics and Mechanism, Part A, (Purich, D. L., ed.) vol 63, pp. 234-257. Orlando: Academic Press.

LiLLO, C. (1984). Circadian rhythmicity of nitrate reductase activity in barley leaves. Physiol. Plant. 61, 219-223.

Lillo, C. \& Ruoff, P. (1984). A minimal oscillator model of light-induced circadian rhythms in leaves of barley. Physiol. Plant. 62, 589-592.

LindBerg, R. G. \& HAYDEN, P. (1974). Entrainment by temperature cycles in the little pocket mouse. Chronobiologia 1, 356-361.

Mohsenzadeh, S., Xu, C., Fracella, F. \& Rensing, L. (1994). Heat shock inhibits and activates different protein degradation pathways and proteinase activities in Neurospora crassa. FEMS Microbiology Letters 124, 215-224.

Murray, J. D. (1989). Mathematical Biology, ch. 3, Berlin: Springer-Verlag.

NaKashima, H. (1987). Comparison of phase shifting by temperature of wild type Neurospora crassa and the clock mutant frq-7. J. Interdiscipl. Cycle Res. 18, 1-8.

Nuus, D., Sulzman, F. M. \& Hastings, J. W. (1974). Membrane model for the circadian clock. Nature 248, 116-120.

Oltmanns, O. (1960). Über den Einfluß der Temperatur auf die endogene Tagesrhythmik und die Blühinduktion bei der Kurztagspflanze Kalanchoe blossfeldiana, Planta 54, 233-264.
Pavlidis, T., Zimmerman, W. F. \& Osborn, J. (1968). A mathematical model for the temperature effects on circadian rhythms. J. theor. Biol. 18, 210-221.

Pittendrigh, C. S. \& Caldarola, P. C. (1973). General homeostasis of the frequency of circadian oscillations. Proc. Nat. Acad. Sci. U.S.A. 70, 2697-2701.

Prigogine, I. \& Lefever, R. (1968). Symmetry breaking instabilities in dissipative systems, J. Chem. Phys. 48, 1695-1700.

Rensing, L., Bos, A., Kroeger, J. \& Cornelius, G. (1987). Possible link between circadian rhythm and heat shock response in Neurospora crassa, Chronobiol. Int., 4, 543-549.

Rensing, L. \& Schill, W. (1985). Perturbation by single and double pulses as analytical tool for analysing oscillatory mechanisms, In: Temporal Order (Rensing, L. \& Jaeger, N. I., eds) pp. 226-231. Berlin: Springer-Berlag.

Rensing, L. \& Schill, W. (1987). Perturbations of cellular circadian rhythms by light and temperature In: Temporal Disorder in Human Oscillatory Systems (Rensing, L., an der Heiden, U. \& Mackey, M. C., eds) pp. 233-245. Berlin: Springer-Verlag.

Rensing, L., Kallies, A., Gebauer, G. \& Mohsenzadeh, S. (1995). The effects of temperature change on the circadian clock of Naurospora In: Circadian Clocks and Their Adjustment (Waterhouse, J. \& Redfern, P., eds) pp. 353-370. Ciba Symposium No. 7.

RössLER, O. E. (1975). Steps toward a temperature-compensated homogeneous chemical clock, In: San Diego Biomedical Symposium, pp. 99-104. San Diego.

RuOFF, P. (1992). Introducing temperature-compensation in any reaction kinetic oscillator model. J. Interdiscipl. Cycle Res. $\mathbf{2 3}$, 92-99.

RuOFF, P. (1994). General homeostasis in period and temperaturecompensated chemical clock mutants formed by random selection conditions, Naturwissenschaften, 81, 456-459.

RuOFF, P. (1995). Antagonistic balance in the Oregonator: About the possibility of temperature-compensation in the BelousovZhabotinsky reaction, Physica D, 84, 204-211.

SinHA, S. (1981). A linear oscillator model for circadian rhythms: Implications for phase response curves, Chronobiologica $\mathbf{8}$, 377-391.

SinHA, S. (1983). Are circadian oscillators structurally unstable? J. theor. Biol. 101, 401-414.

TaYlor, W., Krasnow, R., Dunlap, J. C., Broda, H. \& Hastings, J. W. (1982). Critical pulses of anisomycin drive the circadian oscillator in Gonyaulax polyhedra towards the singularity. J. Comp. Physiol. 148, 11-25.

Thompson, G. A. JR. (1980). Control of membrane fluidity, Clifton: Humana.

TokuRA, H. \& Oishi, T. (1985). Effect of Temperature Cycles in Djungarian Hamsters, Comp. Biochem. Physiol. A, 81A, 271-275.

Winfree, A. T. (1980). The Geometry of Biological Time. New York: Springer-Verlag.

Zeng, H., Hardin, P. E. \& Rosbash, M. (1994). Constitutive overexpression of the Drosophila period protein inhibits period mRNA cycling, EMBO J., 13, 3590-3598.

Zimmerman, W. F., Pittendrigh, C. S. \& Pavlidis, T. (1968). Temperature compensation of the circadian oscillation in Drosophila pseudoobscura and its entrainment by temperature cycles. J. Insect Physiol. 14, 669-684. 